\title{
A counter-example to a recent version of the Penrose conjecture
}

\author{
Alberto Carrasco and Marc Mars \\ Dept. Física Fundamental, Universidad de Salamanca, Plaza de la Merced s/n, 37008 \\ Salamanca, Spain \\ E-mail: acf@usal.es and marc@usal.es
}

\begin{abstract}
By considering suitable axially symmetric slices on the Kruskal spacetime, we construct a counterexample to a recent version of the Penrose inequality in terms of so-called generalized apparent horizons.
\end{abstract}

PACS Numbers: 04.20.q, 04.20.Cv, 04.70.Bw, 02.40.k, 02.40.Vh

\section{Introduction}

In a serious attempt [1] to prove the Penrose inequality in the general case, Bray and Khuri were led to conjecture a new version of the Penrose inequality in terms of so-called generalized apparent horizons. In fact, they proved that if a certain system of PDEs admit solutions with the right boundary behaviour, then such a Penrose inequality follows. In this paper we show that this inequality cannot be true in general by finding slices of the Kruskal spacetime (i.e. the maximally extended Schwarzschild spacetime) for which the outermost generalized apparent horizon has area strictly larger than $16 \pi M^{2}$, where $M$ is the ADM mass of the spacetime. We start with a brief discussion on the Penrose inequality, with the aim of putting the Bray and Khuri proposal into context (see 2] for further details) and then show that there exist slices of Kruskal for which this inequality is violated. For the systems of PDEs proposed in [1], this means that a general existence theory cannot be expected with boundary conditions compatible with generalized apparent horizons. However, simpler boundary conditions (e.g. compatible with future and past apparent horizons) are not ruled out. This may in fact simplify the analysis of these equations.

Penrose [3] noticed that the total mass of a spacetime containing black holes that settle down to a stationary state must satisfy the inequality

$$
M_{A D M} \geq \sqrt{\frac{|\mathcal{H}|}{16 \pi}},
$$

where $|\mathcal{H}|$ is the area of the event horizon at one instant of time. Moreover, assuming the matter contents to satisfy the dominant energy condition and combining (11) with cosmic 
censorship, Penrose observed that new inequalities similar to (1) follow, where the righthand side is replaced by the area of certain surfaces which can be defined independently of the future evolution of the spacetime (in contrast to the event horizon). This type of inequalities are collectively termed Penrose inequalities. Their main interest is two-fold. Firstly, they provide strengthenings of the positive mass theorem. Secondly, since cosmic censorship is the basic physical ingredient supporting their validity, a direct proof of the inequality would give rather strong indirect support for the cosmic censorship conjecture.

There are several versions of the Penrose inequality. Typically, one considers closed (i.e. compact and without boundary) surfaces $S$ embedded in a spacelike and asymptotically flat hypersurface $\Sigma$, which are bounding, i.e. such that $S$ divides $\Sigma$ into two open regions. The region containing the asymptotically flat end is called "exterior", while it complementary is the "interior" of $S$. Given two bounding surfaces $S_{1}$ and $S_{2}$, we say that $S_{1}$ encloses $S_{2}$ provided the exterior of $S_{2}$ contains the exterior of $S_{1}$. We denote by $\theta^{+}$and $\theta^{-}$the null expansions along the outer and inner future null directions respectively. A surface is called weakly outer trapped iff $\theta^{+} \leq 0$ and marginally outer trapped surfaces (MOTS) if $\theta^{+}=0$. In terms of the initial data set $\left(\Sigma, g_{i j}, K_{i j}\right)$, we have $\theta^{ \pm}=p \pm q$, where $p$ is the mean curvature of $S \subset\left(\Sigma, g_{i j}\right)$ with respect to the outer unit normal $\vec{m}$ and $q$ is the trace of the pull-back of the second fundamental form $K_{i j}$ onto $S$. The union of the interiors of all weakly outer trapped surfaces defines the so-called outer trapped set $\mathcal{T}^{+}$in $\Sigma$. The boundary of this set is a smooth MOTS [4].

The standard version of the Penrose inequality reads

$$
M \geq \sqrt{\frac{A_{\min }\left(\partial \mathcal{T}^{+}\right)}{16 \pi}},
$$

where $A_{\min }\left(\partial \mathcal{T}^{+}\right)$is the minimal area necessary to enclose $\partial \mathcal{T}^{+}$. The need of taking this minimal area enclosure comes from the fact that, under cosmic censorship, we know that the event horizon encloses $\partial \mathcal{T}^{+}$. However, the former could still have less area than $\partial \mathcal{T}^{+}$and, since its location is undetermined, the minimum of area of all enclosing surfaces should be taken. Inequality (2) also has a rigidity statement, namely that equality implies that $\left(\Sigma, g_{i j}, K_{i j}\right)$ is a slice of the Kruskal spacetime.

By reversing the time orientation, the same argument yields (2) with $\partial \mathcal{T}^{+}$replaced by $\partial \mathcal{T}^{-}$, where $\mathcal{T}^{-}$is the union of the interiors of all bounding surfaces satisfying $\theta^{-} \geq 0$. In general, neither $\partial \mathcal{T}^{+}$encloses $\partial \mathcal{T}^{-}$nor vice versa. In the time-symmetric case $K_{i j}=0$, the inequality simplifies because $\mathcal{T}^{+}=\mathcal{T}^{-}$and its boundary is the outermost minimal surface (i.e. a minimal surface enclosing any other bounding minimal surface in $\Sigma$ ), and hence its own minimal area enclosure. The inequality in this case is called Riemannian Penrose inequality and it has been proven for one black hole in [5] and in full generality in [6] using a different method. In the non-time symmetric case, (2) is not invariant under time reversals. Moreover, the minimal area enclosure of a given surface $S$ can be a rather complicated object typically consisting of portions of $S$ together with portions of minimal surfaces (i.e. surfaces with $p=0$ ) outside of $S$. This complicates the problem substantially. This has led several authors to propose simpler looking versions 
of the inequality, even if they are not directly supported by cosmic censorship. Two such extensions are

$$
M \geq \sqrt{\frac{A_{\min }\left(\partial\left(\mathcal{T}^{+} \cup \mathcal{T}^{-}\right)\right)}{16 \pi}}, \quad M \geq \sqrt{\frac{\left|\partial\left(\mathcal{T}^{+} \cup \mathcal{T}^{-}\right)\right|}{16 \pi}},
$$

where $|S|$ denotes the area of $S$ (cf. [7]). These inequalities are immediately stronger than (2) and have the advantage of being invariant under time reversals. The second avoids even the use of minimal area enclosures. Neither version is supported by cosmic censorship and at present there is little evidence for their validity. However, both reduce to the standard version in the Riemannian case and both hold in spherical symmetry. No counterexamples are known either. It would be interesting to have either stronger support for them, or else to find a counterexample.

Recently, Bray and Khuri proposed a new method to approach the general (i.e. non time-symmetric) Penrose inequality. The basic idea was to modify the Jang equation [8], 9] so that the product manifold $\Sigma \times \mathbb{R}$ used to construct the graphs which define the Jang equation is endowed with a warped type metric of the form $-\varphi^{2} d t^{2}+g$ instead of the product metric. The aim was to reduce the general Penrose inequality to the Riemannian Penrose inequality on the graph manifold. A discussion on the type of divergences that could possibly occur for the generalized Jang equation led the authors to consider a new type of bounding surfaces called generalized trapped surfaces and generalized apparent horizons, which are defined, respectively, by $p \leq|q|$ and $p=|q|$. This type of surfaces have very interesting properties. The most notable one is that, on any asymptotically flat initial data set containing at least one generalized trapped surface, there is always a unique $C^{2, \alpha}$ outermost generalized apparent horizon $S_{\text {out }}$ [10]. Moreover, this surface has smaller area than any other surface enclosing it [10]. Bray and Khuri's version of the Penrose inequality reads

$$
M \geq \sqrt{\frac{\left|S_{\text {out }}\right|}{16 \pi}}
$$

This inequality has several remarkable properties that makes it very appealing [1]. First of all, the definition of generalized apparent horizon, and hence the corresponding Penrose inequality, is insensitive to time reversals. Moreover, there is no need of taking the minimal area enclosure of $S_{\text {out }}$, as this surface has less area than any of its enclosures. Since MOTS are automatically generalized trapped surfaces, $S_{\text {out }}$ encloses the outermost MOTS $\partial \mathcal{T}^{+}$. Thus, (44) is stronger than (2) and its proof would also establish the standard version of the Penrose inequality. Moreover, Khuri has proven [1] that no generalized trapped surfaces exist in Minkowski, which is a necessary condition for the validity of (4). Another interesting property of this version, and one of its motivations discussed in [1], is that the equality case in (4) covers a larger number of slices of Kruskal than the equality case in (2). Recall that the rigidity statement of any version of the Penrose inequality asserts that equality implies that $\left(\Sigma, g_{i j}, K_{i j}\right)$ is a hypersurface of Kruskal. However, which slices of Kruskal satisfy the equality case may depend on the version under consideration. The more slices have this property, the more accurate the 
version can be considered. For any slice $\Sigma$ of Kruskal we can define its exterior region $\Sigma^{+}$as the intersection of $\Sigma$ with the domain of outer communications. Bray and Khuri noticed that whenever $\partial \Sigma^{+}$intersects both the black hole and the white hole event horizons, then the standard version (2) gives, in fact, a strict inequality. Although (4) does not give equality for all slices of Kruskal, it does so in all cases where the boundary of $\Sigma^{+}$is a $C^{2, \alpha}$ surface (provided this boundary - which is a generalized apparent horizon in this case - is, in fact, the outermost such horizon). It follows that version (4) contains more cases of equality than (2) and is therefore more accurate. It should be stressed that the second inequality in (3) gives equality for all slices of Kruskal, so in this sense it would be optimal.

Despite its appealing properties, (41) is not directly supported by cosmic censorship. The reason is that the outermost generalized apparent horizon need not always lie inside the event horizon. A simple example [2] is given by a slice $\Sigma$ of Kruskal such that $\partial \mathcal{T}^{+}$ (which corresponds to the intersection of $\Sigma$ with the black hole event horizon) and $\partial \mathcal{T}^{-}$ (the intersection $\Sigma$ with the white hole horizon) meet transversally. Since both surfaces are generalized trapped surfaces, Eichmair's theorem [10] implies that there must exist a unique $C^{2, \alpha}$ outermost generalized apparent horizon enclosing both. This surface must therefore penetrate into the exterior region somewhere, as claimed. It becomes natural to study the outermost generalized apparent horizon in slices of this type in order to check whether (4) holds or not. The result is that there are examples for which (4) turns out to be violated. More precisely, in this paper we prove the following statement.

Theorem 1 In the Kruskal spacetime with mass $M>0$, there exist asymptotically flat, spacelike hypersurfaces with an outermost generalized apparent horizon $S_{\text {out }}$ satisfying $\left|S_{\text {out }}\right|>16 \pi M^{2}$.

\section{Construction of the counterexample.}

Let us consider the Kruskal spacetime of mass $M>0$ with metric

$$
d s^{2}=\frac{32 M^{3}}{r} e^{-r / 2 M} d \hat{u} d \hat{v}+r^{2}\left(d \theta^{2}+\sin \theta^{2} d \phi^{2}\right),
$$

where $r(\hat{u} \hat{v})$ solves the implicit equation $\hat{u} \hat{v}=e^{r / 2 M}(r-2 M) /(2 M)$. In this metric $\partial_{\hat{v}}$ is future-directed and $\partial_{\hat{u}}$ is past-directed. The region $\{\hat{u}>0, \hat{v}>0\}$ defines the domain of outer communications and $\{\hat{u}=0\},\{\hat{v}=0\}$ define, respectively, the black hole and white hole event horizons. Consider the one-parameter family of axially-symmetric embedded hypersurfaces $\Sigma_{\epsilon}=\mathbb{R} \times S^{2}$, with intrinsic coordinates $\hat{y} \in \mathbb{R}, x \in[-1,1]$, $\phi \in[0,2 \pi]$, defined by the embedding

$$
\Sigma_{\epsilon} \equiv\{\hat{u}=\hat{y}-\epsilon x, \hat{v}=\hat{y}+\epsilon x, \cos \theta=x, \phi=\phi\} .
$$

It is easy to check that these hypesurfaces are well-defined, smooth and asymptotically flat for all $|\epsilon|<1$. Morever, it is straightforward to show that $\Sigma_{\epsilon}$ is spacelike everywhere for $|\epsilon|$ small enough. The discrete isometry of the Kruskal spacetime defined by $\{\hat{u}, \hat{v}\} \rightarrow\{\hat{v}, \hat{u}\}$ implies that under reflection with respect to the equatorial plane, 
i.e. $(\hat{y}, x, \phi) \rightarrow(\hat{y},-x, \phi)$, the induced metric of $\Sigma_{\epsilon}$ remains invariant, while the second fundamental form of $\Sigma_{\epsilon}$ changes sign. The exterior region $\Sigma_{\epsilon}^{+}$of $\Sigma_{\epsilon}$ is given by $\{\hat{y}-|\epsilon x|>0\}$. For $\epsilon \neq 0, \partial \Sigma_{\epsilon}^{+}$is composed by a portion of the black hole event horizon and a portion of the white hole event horizon. Moreover, $\partial \mathcal{T}^{+}$is given by $\{\hat{y}-\epsilon x=0\}$, while $\partial \mathcal{T}^{-}$is $\{\hat{y}+\epsilon x=0\}$ so that these surfaces intersect transversally on the circumference $\{\hat{y}=0, x=0\}$ provided $\epsilon \neq 0$. By Eichmair's theorem, there exists a $C^{2, \alpha}$ outermost generalized apparent horizon containing both $\partial \mathcal{T}^{+}$and $\partial \mathcal{T}^{-}$. Uniqueness implies that this surface must be axially symmetric and have equatorial symmetry. In order to locate it, we proceed in two steps. First we will show that an axial and equatorially symmetric generalized apparent horizon of spherical topology and lying in a sufficiently small neighbourhood of $\{\hat{y}=0\}$ exists, provided $\epsilon$ is small enough. We will also determine its embedding function to first order in $\epsilon$. In the second step we prove that this surface is either the outermost generalized apparent horizon, or else, it has smaller area than any other generalized apparent horizon in $\Sigma_{\epsilon}$ enclosing it.

Thus, we consider surfaces of spherical topology defined by embedding functions $\{\hat{y}=y(x, \epsilon), x=x, \phi=\phi\}$ and satisfying $y(-x, \epsilon)=y(x, \epsilon)$. Since the outermost generalized apparent horizon is known to be $C^{2, \alpha}$ it is natural to consider the spaces of functions $U^{m, \alpha} \equiv\left\{y \in C^{m, \alpha}\left(S^{2}\right): \partial_{\phi} y=0, y(-x)=y(x)\right\}$, i.e. the spaces of $m$-times differentiable functions on the unit sphere, with Hölder continuous $m$-th derivatives with exponent $\alpha \in(0,1)$ and invariant under the axial Killing vector on $S^{2}$ and under reflection about the equatorial plane. Each space $U^{m, \alpha}$ is a closed subset of the Banach space $C^{m, \alpha}\left(S^{2}\right)$ and hence a Banach space itself. Let $I \subset \mathbb{R}$ be the closed interval where $\epsilon$ takes values. For each function $y \in U^{2, \alpha}$ the expression $p-|q|$ defines a non-linear map $f: U^{2, \alpha} \times I \rightarrow U^{0, \alpha}$. Thus, we are looking for the outermost of the solutions $y \in U^{2, \alpha}$ of the equation $f=0$. We know that when $\epsilon=0$, the hypersurface $\Sigma_{\epsilon}$ is totally geodesic, which implies $q=0$ for any surface on it. Consequently, all generalized apparent horizons on $\Sigma_{\epsilon=0}$ satisfy $p=0$ and are, in fact, minimal surfaces. The only closed minimal surface in $\Sigma_{\epsilon=0}$ is the bifurcation surface $\{\hat{u}=0, \hat{v}=0\}$. Thus, the equation $f(y, \epsilon)=0$ has $y=0$ as the unique solution when $\epsilon=0$. It becomes natural to use the implicit function theorem for Banach spaces to show that there exists a unique solution $y \in U^{2, \alpha}$ of $f=0$ in a neighbourhood of $y=0$ for $\epsilon$ small enough. The implicit function theorem requires the operator $f$ to have a continuous Fréchet derivative and the partial derivative $\left.D_{y} f\right|_{(y=0, \epsilon=0)}$ to be an isomorphism. The appearance of an absolute value in $|q|$ makes the Fréchet derivative of $f$ potentially discontinuous [12]. However, the problem can be solved considering a suitable modification of $f$. Since the details are somewhat technical, we postpone the proof of this fact to an Appendix, where we establish the followig proposition.

Proposition 1 There exists a neighborhood $\tilde{I} \subset I$ of $\epsilon=0$ such that $f(y, \epsilon)=0$ admits a solution $y(x, \epsilon) \in C^{2, \alpha}\left(S^{2}\right)$ for all $\epsilon \in \tilde{I}$. Moreover, $y(x, \epsilon)$ is $C^{1}$ in $\epsilon$ and satisfies $y(x, \epsilon=0)=0$. 
Let us denote by $S_{\epsilon}$ the surface defined by this solution. The proposition above implies that we can expand $y(x, \epsilon)=Y_{1}(x) \epsilon+o(\epsilon)$. By linearizing the PDE $f(y, \epsilon)=0$ in $\epsilon$, it turns out that $Y_{1}$ satisfies the linear equation $L\left(Y_{1}(x)\right)=3|x|$, where $L(z(x)) \equiv$ $-\left(1-x^{2}\right) \ddot{z}+2 x \dot{z}+z$. The right hand side of this equation corresponds (except for a positive multiplicative constant) to the linearization of $|q|$. By decomposing into Legendre polynomials $P_{n}(x)$, it is easy to show that the unique solution of this equation is

$$
Y_{1}(x)=\frac{3}{2}+\sum_{n=1}^{\infty} a_{2 n} P_{2 n}(x), \quad a_{2 n}=\frac{3(4 n+1)(-1)^{n+1}}{[2 n(2 n+1)+1] 2^{2 n}} \frac{(2 n-2) !}{(n-1) !(n+1) !},
$$

where convergence is in $L^{2}$. This expression allows us to compute the area of $S_{\epsilon}$ at once. After a straightforward calculation we find

$$
\left|S_{\epsilon}\right|=16 \pi M^{2}+\frac{8 \pi M^{2} \epsilon^{2}}{e}\left(5+4 \sum_{n=1}^{\infty} \frac{2 n(2 n+1)+1}{4 n+1} a_{2 n}^{2}\right)+O\left(\epsilon^{3}\right) .
$$

Since the second summand is strictly positive, it follows that $\left|S_{\epsilon}\right|>16 \pi M^{2}$. If we could show that $S_{\epsilon}$ is the outermost generalized apparent horizon, we would have a counterexample of (4). Before turning into this point, however, let us give an alternative argument to show that the area increases. This will shed some light into the underlying reason why the area of $S_{\epsilon}$ is larger than $16 \pi M^{2}$.

To that aim, let us now use coordinates $\{\hat{u}, x, \phi\}$ in $\Sigma_{\epsilon}$. Then, the embedding of $\Sigma_{\epsilon}$ becomes $\Sigma_{\epsilon} \equiv\{\hat{u}, \hat{v}=\hat{u}+2 \epsilon x, x, \phi\}$, and the corresponding embedding in $\Sigma_{\epsilon}$ for the surfaces $S_{\epsilon}$ is $S_{\epsilon}=\{\hat{u}=u(x, \epsilon), x, \phi\}$. Again, $u$ admits an expansion $u=U_{1}(x) \epsilon+o(\epsilon)$. The relationship between $U_{1}$ and $Y_{1}$ is simply $Y_{1}=U_{1}+x$. It follows that $U_{1}$ satisfies $L\left(U_{1}(x)\right)=3(|x|-x)$. Similarly, if we take $\{\hat{v}, x, \phi\}$ as coordinates for $\Sigma_{\epsilon}$, then the embedding of $S_{\epsilon}$ reads $\hat{v}=V_{1}(x) \epsilon+o(\epsilon)$, with $V_{1}$ satisfying the equation $L\left(V_{1}(x)\right)=3(|x|+x)$. Thus, $L\left(U_{1}(x)\right) \geq 0$ and $L\left(V_{1}(x)\right) \geq 0$ and neither of them is identically zero. Since $L$ is an elliptic operator with positive zero order term, we can use the maximum principle to conclude that $U_{1}(x)>0$ and $V_{1}(x)>0$ everywhere. Geometrically, this means that $S_{\epsilon}$ lies fully in $\Sigma_{\epsilon}^{+}$for $\epsilon$ small enough. In fact, the maximum principle applied to $L\left(Y_{1}\right)=3|x|$ also implies $Y_{1}>0$. This will be used below.

We can now view $S_{\epsilon}$ as a first order spacetime variation of the bifurcation surface. The variation vector $\partial_{\epsilon}$ is defined as the tangent vector to the curve generated when a point with fixed coordinates $\{x, \phi\}$ in $S_{\epsilon}$ moves as $\epsilon$ varies. By the argument above, this vector is spacelike everywhere on the unperturbed surface $S_{\epsilon=0}$. If we do a Taylor expansion of $\left|S_{\epsilon}\right|$ around $\epsilon=0$, we see that the zero order term is $\left|S_{\epsilon=0}\right|=16 \pi M^{2}$, as this is the area of the bifurcation surface. The bifurcation surface is totally geodesic so that, in particular, its mean curvature vector vanishes. Consequently, the linear term in the expansion is identically zero as a consequence of the first variation of area

$$
\frac{d\left|S_{\epsilon}\right|}{d \epsilon}=\int_{S_{\epsilon}}\left(\vec{H}_{S_{\epsilon}}, \partial_{\epsilon}\right) \boldsymbol{\eta}_{\boldsymbol{S}_{\epsilon}}
$$


where $\vec{H}_{S_{\epsilon}}$ is the spacetime mean curvature vector of $S_{\epsilon}$ and $($,$) denotes scalar product$ with the spacetime metric. For the second order term in the expansion, we take the derivative of (5) with respect to $\epsilon$ and evaluate at $\epsilon=0$. A simple computation gives

$$
\left.\frac{d^{2}\left|S_{\epsilon}\right|}{d \epsilon^{2}}\right|_{\epsilon=0}=\frac{16 \pi M^{2}}{e} \int_{-1}^{1}\left[U_{1}(x) L\left(V_{1}(x)\right)+V_{1}(x) L\left(U_{1}(x)\right)\right] d x .
$$

Since $U_{1}$ and $V_{1}$ are strictly positive and $L\left(U_{1}(x)\right), L\left(V_{1}(x)\right)$ are non-negative and not identically zero, it follows $\left.\frac{d^{2}\left|S_{\epsilon}\right|}{d \epsilon^{2}}\right|_{\epsilon=0}>0$ and hence that the area of $S_{\epsilon}$ is larger than $16 \pi M^{2}$ for small $\epsilon$. The fact that the area increases is therefore a consequence of the fact that the second order variation of area turns out to be strictly positive along the direction joining the bifurcation surface with $S_{\epsilon}$, and, in turn, this is tied to the fact that $L\left(U_{1}\right)$ and $L\left(V_{1}\right)$ have a sign. The right hand sides of these operators are (except for a constant) the linearization of $|q| \pm q$ and these objects are obviously non-negative in all cases. We conclude, therefore, that the fact that the area of $S_{\epsilon}$ is larger than $16 \pi M^{2}$ is closely related to the defining equation $p=|q|$. It follows that the increase of area is a robust property which does not depend strongly on the choice of hypersurfaces $\Sigma_{\epsilon}$ that we have made. In fact, had we chosen hypersurfaces $\Sigma_{\epsilon} \equiv\{u=y-\epsilon \beta(x), v=y+\epsilon \beta(x), \cos \theta=x, \phi=\phi\}$, the corresponding equations would have been $L\left(U_{1}(x)\right)=|L(\beta(x))|-L(\beta(x))$ and $L\left(V_{1}(x)\right)=|L(\beta(x))|+L(\beta(x))$. The same conclusions would follow provided the right hand sides are not identically zero.

Having shown that $\left|S_{\epsilon}\right|>16 \pi M^{2}$ for $\epsilon \neq 0$ small enough, the next step is to analyze whether $S_{\epsilon}$ is the outermost generalized apparent horizon or not. In fact, in order to have a counterexample of (4) we only need to make sure that no generalized apparent horizon with less area than $S_{\epsilon}$ and enclosing $S_{\epsilon}$ exists in $\Sigma_{\epsilon}$. We will argue by contradiction. Let $\hat{S}_{\epsilon}$ be a generalized apparent horizon enclosing $S_{\epsilon}$ and with $\left|\hat{S}_{\epsilon}\right|<\left|S_{\epsilon}\right|$. Then, since $S_{\epsilon}$ is not area outer minimizing, its minimal area enclosure $S_{\epsilon}^{\prime}$ does not coincide with $S_{\epsilon}$. Now, two possibilities arise: (i) either $S_{\epsilon}^{\prime}$ lies completely outside $S_{\epsilon}$, or (ii) it coincides with $S_{\epsilon}$ on a closed subset $K_{\epsilon}$, while the complement $S_{\epsilon}^{\prime} \backslash K_{\epsilon}$ (which is non-empty) has vanishing mean curvature $p$ everywhere. To exclude case (i), consider the foliation of $\Sigma_{\epsilon}$ defined by the surfaces $\left\{\hat{y}=y_{0}, x, \phi\right\}$, where $y_{0}$ is a constant. A direct computation shows that the mean curvature $p_{y_{0}}$ of these surfaces with respect to the outer normal is positive for all $y_{0}>0$. We noted above that $Y_{1}(x)>0$ everywhere. Thus, for small enough $\epsilon$, the function $y(x, \epsilon)$ is also strictly positive. Since $S_{\epsilon}^{\prime}$ lies fully outside $S_{\epsilon}$, the coordinate function $\hat{y}$ restricted to $S_{\epsilon}^{\prime}$ achieves a positive maximum $y_{\epsilon}$ somewhere. At this point, the two surfaces $S_{\epsilon}^{\prime}$ and $\left\{\hat{y}=y_{\epsilon}\right\}$ meet tangentially, with $S_{\epsilon}^{\prime}$ lying fully inside $\left\{\hat{y}=y_{\epsilon}\right\}$. This is a contradiction to the maximum principle for minimal surfaces. It only remains to deal with case (ii). The same argument above shows that the coordinate function $\hat{y}$ restricted to $S_{\epsilon}^{\prime} \backslash K_{\epsilon}$ cannot reach a local maximum. It follows that the range of variation of $\hat{y}$ restricted to $S_{\epsilon}^{\prime}$ is contained in the range of variation of $\hat{y}$ restricted to $S_{\epsilon}$. Since $\max _{S_{\epsilon}} \hat{y}-\min _{S_{\epsilon}} \hat{y}=O(\epsilon)$, it follows that we can regard $S_{\epsilon}^{\prime}$ as an outward variation of $S_{\epsilon}$ of order $\epsilon$ when $\epsilon$ is taken small enough. The corresponding variation 
vector field $\vec{\xi}$ can be taken orthogonal to $S_{\epsilon}$ without loss of generality, i.e. $\vec{\xi}=\xi \vec{m}$, where $\vec{m}$ is the outward unit normal to $S_{\epsilon}$. The function $\xi$ vanishes on $K_{\epsilon}$ and is positive in its complement $U_{\epsilon} \equiv S_{\epsilon} \backslash K_{\epsilon}$. Expanding to second order and using the first and second variation of area (see e.g. [13]) gives

$$
\begin{aligned}
\left|S_{\epsilon}^{\prime}\right|=\mid & S_{\epsilon} \mid+\epsilon \int_{U_{\epsilon}} p_{S_{\epsilon}} \xi \boldsymbol{\eta}_{\boldsymbol{S}_{\epsilon}}+ \\
& +\frac{\epsilon^{2}}{2} \int_{U_{\epsilon}}\left(\left|\nabla_{S_{\epsilon}} \xi\right|^{2}+\frac{\xi^{2}}{2}\left(R^{S_{\epsilon}}-R^{\Sigma_{\epsilon}}-\left|A_{S_{\epsilon}}\right|^{2}+p_{S_{\epsilon}}^{2}\right)+p_{S_{\epsilon}} \frac{d \xi}{d \epsilon}\right) \boldsymbol{\eta}_{S_{\epsilon}}+O\left(\epsilon^{3}\right),
\end{aligned}
$$

where $\nabla_{S_{\epsilon}}, R^{S_{\epsilon}}$ and $A_{S_{\epsilon}}$ are, respectively, the gradient, scalar curvature and second fundamental form of $S^{\epsilon}$, and $R^{\Sigma_{\epsilon}}$ is the scalar curvature of $\Sigma_{\epsilon}$. Now, the mean curvature $p_{S_{\epsilon}}$ of $S_{\epsilon}$ reads $p_{S_{\epsilon}}=\frac{3 \epsilon}{M \sqrt{e}}|x|+o(\epsilon)$ and both $R^{\Sigma_{\epsilon}}$ and $A_{S_{\epsilon}}$ are of order $\epsilon$ (because $\Sigma_{\epsilon=0}$ has vanishing scalar curvature and $S_{\epsilon=0}$ is totally geodesic). Moreover $R^{S_{\epsilon}}=1 /\left(2 M^{2}\right)+O(\epsilon)$. Thus,

$$
\left|S_{\epsilon}^{\prime}\right|=\left|S_{\epsilon}\right|+\epsilon^{2}\left\{\int_{U_{\epsilon}}\left[\frac{3|x| \xi}{M \sqrt{e}}+\left(\frac{\left|\nabla_{S_{\epsilon}} \xi\right|^{2}}{2}+\frac{\xi^{2}}{8 M^{2}}\right)\right] \boldsymbol{\eta}_{\boldsymbol{S}_{\epsilon}}\right\}+O\left(\epsilon^{3}\right) .
$$

It follows that, for small enough $\epsilon$, the area of $S_{\epsilon}^{\prime}$ is larger than $S_{\epsilon}$ contrarily to our assumption. This proves Theorem 1 and, therefore, the existence of counterexamples to the version (4) of the Penrose inequality.

A final remark is in order. As already mentioned at the beginning, the existence of this counterexample does not invalidate the approach suggested by Bray and Khuri based on the generalized Jang equation to study the general Penrose inequality. It means, however, that the emphasis should not be put on generalized apparent horizons. It may be that the approach can serve to prove the standard version (2) as recently discussed in [14. Alternatively, let us note that, since the slice $\Sigma_{\epsilon}$ lies in the Kruskal spacetime, it is immediate that the generalized Jang equation admits solutions on $\Sigma_{\epsilon}$ which blow up a non-empty subset of $\partial \Sigma_{\epsilon}^{+}$and blown down on another non-empty subset of this boundary, provided the warping function $\varphi^{2}$ is chosen to be $\varphi^{2}=1-2 M /\left.r\right|_{\Sigma_{\epsilon}}$. The induced metric on the graph is then isometric to the Schwarzschild metric $h=$ $\frac{d r^{2}}{1-2 M / r}+r^{2} d \Omega^{2}$ restricted to $r>2 M$. The boundary is therefore a minimal surface (in fact, totally geodesic) despite the fact that $\partial \Sigma_{\epsilon}$ is not smooth in $\Sigma_{\epsilon}$. This property turns out to be general for any slice $\Sigma$ in an asymptotically flat spacetime with a hypersurface orthogonal Killing vector $\vec{\eta}$ which is timelike at infinity [15]. More precisely, assuming $(\Sigma, g, K)$ to be analytic and defining $\Sigma^{+}$to be the largest connected subset of $\Sigma$ containing the asymptotic end such that $\vec{\eta}$ is timelike, the so-called quotient metric $h$ can be defined on $\Sigma^{+}$. In general, $\partial \Sigma^{+}$is not smooth. However, there exists a differentiable structure on $\overline{\Sigma^{+}}$such that $\partial \Sigma^{+}$is smooth and either lies at infinity with respect to $h$, or else, this metric extends smoothly to the boundary, which becomes a totally geodesic submanifold [15]. This fact seems to suggest that the PDE method of Bray and Khuri might be suitable even for approaching the second inequality in (3). At present, however, this remains rather speculative. 


\section{Appendix A. Proof of Proposition 1}

Firstly, let us consider surfaces in $\Sigma_{\epsilon}$ defined by $\{\hat{y}=y(x, \epsilon), x, \phi\}$ such that the embedding function has the form $y=\epsilon Y$, where $Y \in U^{2, \alpha}$. An explicit computation of the mean curvature $p$ on such surfaces gives $p=\epsilon \mathcal{P}(Y(x), \dot{Y}(x), \ddot{Y}(x), x, \epsilon)$, where dot denotes derivative with respect to $x$ and where $\mathcal{P}: \mathbb{R}^{3} \times[-1,1] \times I \rightarrow \mathbb{R}$ is a smooth (in fact, analytic) function. Similarly $q=\epsilon \mathcal{Q}(Y(x), \dot{Y}(x), x, \epsilon)$, where $\mathcal{Q}: \mathbb{R}^{2} \times[-1,1] \times I \rightarrow \mathbb{R}$ is an analytic function. Moreover, the function $\mathcal{Q}$ has the symmetry $\mathcal{Q}\left(x_{1}, x_{2}, x_{3}, x_{4}\right)=-\mathcal{Q}\left(x_{1},-x_{2},-x_{3}, x_{4}\right)$, which reflects the fact that the extrinsic curvature of $\Sigma_{\epsilon}$ changes sign under a transformation $x \rightarrow-x$. Let us write $P(Y, \epsilon)(x) \equiv \mathcal{P}(Y(x), \dot{Y}(x), \ddot{Y}(x), x, \epsilon)$ and similarly $Q(Y, \epsilon)(x) \equiv \mathcal{Q}(Y(x), \dot{Y}(x), x, \epsilon)$.

Now, instead of $f$, let us consider the functional $F: U^{2, \alpha} \times I \rightarrow U^{0, \alpha}$ defined by $F(Y, \epsilon)=P(Y, \epsilon)-|Q(Y, \epsilon)|$. This functional has the property that, for $\epsilon>0$, the solutions of $F(Y, \epsilon)=0$ correspond exactly to the solutions of $f(y, \epsilon)=0$ via the relation $y=\epsilon Y$. Moreover, the functional $F$ is well-defined for all $\epsilon \in I$, in particular at $\epsilon=0$. Therefore, by proving that $F=0$ admits solutions in a neighbourhood of $\epsilon=0$, we will conclude that $f=0$ admits solutions for $\epsilon>0$ and the solutions will in fact belong to a neighbourhood of $y=0$ since $y=\epsilon Y$.

In order to show that $F$ admits solutions we will use the implicit function theorem. A direct calculation yields $F(Y, \epsilon=0)(x)=c(L(Y)(x)-3|x|)$ where $c$ is the constant $1 /(m \sqrt{e})$ and $L(Y) \equiv-\left(1-x^{2}\right) \ddot{Y}+2 x \dot{Y}+Y$. This operator is an isomorphism between $U^{2, \alpha}$ and $U^{0, \alpha}$. Let $Y_{1} \in U^{2, \alpha}$ be the unique solution of the equation $L(Y)=3|x|$. For later use, we note that $Q\left(Y_{1}, \epsilon=0\right)=-3 c x$. This vanishes only at $x=0$. This is the key property that allows us to prove that $F$ is $C^{1}\left(U^{2, \alpha} \times I\right)$.

The $C^{1}\left(U^{2, \alpha} \times I\right)$ property of the functional $P(Y, \epsilon)$ is standard. More subtle is to show that $|Q|$ is $C^{1}\left(U^{2, \alpha} \times I\right)$ in a suitable neighbourhood of $\left(Y_{1}, \epsilon=0\right)$. Let $r_{0}>0$ and define $\mathcal{V}_{r_{0}}=\left\{(Y, \epsilon) \in U^{2, \alpha} \times I:\left\|\left(Y-Y_{1}, \epsilon\right)\right\|_{U^{2, \alpha} \times I} \leq r_{0}\right\}$. First of all we need to show that $|Q|$ is (Fréchet-)differentiable on $\mathcal{V}_{r_{0}}$, i.e. that for all $(Y, \epsilon) \in \mathcal{V}_{r_{0}}$ there exists a continuous linear mapping $D_{Y, \epsilon}|Q|: U^{2, \alpha} \times I \rightarrow U^{0, \alpha}$ such that, for all $(H, \delta) \in U^{2, \alpha} \times I,|Q(Y+H, \epsilon+\delta)|-|Q(Y, \epsilon)|=D_{Y, \epsilon}|Q|(H, \delta)+R_{Y, \epsilon}(H, \delta)$ where $\left\|R_{Y, \epsilon}(H, \delta)\right\|_{U^{0, \alpha}}=o\left(\|(H, \delta)\|_{U^{2, \alpha} \times I}\right)$. The key observation is that, by choosing $r_{0}$ small enough, we have

$$
|Q(Y, \epsilon)(x)|=-\sigma(x) Q(Y, \epsilon)(x)
$$

where $\sigma(x)$ is the sign function, (i.e. $\sigma(x)=+1$ for $x \geq 0$ and $\sigma(x)=-1$ for $x<0)$. For $x$ away from a neightbourhood of 0 , this is a consequence of the fact that $Q\left(Y_{1}, \epsilon=0\right)=-3 c x$, which is negative for $x>0$ and positive for $x<0$. Taking $r_{0}$ small enough, and using that $\mathcal{Q}$ is a smooth function of their arguments, the same inequalities hold for any $(Y, \epsilon) \in \mathcal{V}_{r_{0}}$. Moreover, the function $Q(Y, \epsilon)(x)$ is odd in $x$, so it passes through zero at $x=0$. Hence, in a small enough neighbourhood of $x=0$, the relation (A.1) holds provided we can prove that $Q(Y, \epsilon)$ is strictly decreasing at $x=0$. But this follows inmediately from the fact that $\left.\frac{d Q\left(Y_{1}, \epsilon=0\right)}{d x}\right|_{x=0}=-3 c$ and $\mathcal{Q}$ is a smooth function of its arguments. 
From its definition, it follows that $Q(Y, \epsilon)(x)$ is $C^{1, \alpha}$ and that the functional $Q_{Y, \epsilon}$ has Fréchet derivative $D_{Y, \epsilon} Q(H, \delta)(x)=A_{Y, \epsilon}(x) H(x)+B_{Y, \epsilon}(x) \dot{H}(x)+C_{Y, \epsilon}(x) \delta$, where $\left.A_{Y, \epsilon}(x) \equiv \partial_{1} \mathcal{Q}\right|_{(Y(x), \dot{Y}(x), x, \epsilon)},\left.B_{Y, \epsilon}(x) \equiv \partial_{2} \mathcal{Q}\right|_{(Y(x), \dot{Y}(x), x, \epsilon)}$ and $\left.C_{Y, \epsilon}(x) \equiv \partial_{4} \mathcal{Q}\right|_{(Y(x), \dot{Y}(x), x, \epsilon)}$. We note that these three functions are $C^{1, \alpha}$ and that $A_{Y, \epsilon}, C_{Y, \epsilon}$ are odd, while $B_{Y, \epsilon}$ is even (as a consequence of the symmetries of $\mathcal{Q}$ ). Defining the linear map $D_{Y, \epsilon}|Q|(H, \delta) \equiv$ $-\sigma\left(A_{Y, \epsilon} H+B_{Y, \epsilon} \dot{H}+C_{Y, \epsilon} \delta\right)$, it follows from (A.1) that $|Q(Y+H, \epsilon+\delta)|-|Q(Y, \epsilon)|=$ $D_{Y, \epsilon}|Q|(H, \delta)+R_{Y, \epsilon}(H, \delta)$ with $\|R(H, \delta)\|_{U^{0, \alpha}}=o\left(\|(H, \delta)\|_{U^{2, \alpha} \times I}\right)$. In order to conclude that $D_{Y, \epsilon}|Q|$ is the derivative of $|Q(Y, \epsilon)|$, we only need to check that, it is (i) welldefined (i.e. that its image belongs to $U^{0, \alpha}$ ) and (ii) that it is continuous, i.e. that $\left\|D_{Y, \epsilon}|Q|(H, \delta)\right\|_{U^{0, \alpha}}<C\|(H, \delta)\|_{U^{2, \alpha} \times I}$ for some constant $C$. To show (i), the most difficult term is $-\sigma B_{Y, \epsilon} \dot{H}$, because $B_{Y, \epsilon}(x)$ is even and need not vanish at $x=0$. However $\dot{H}$ is an odd function, and hence $-\sigma B_{Y, \epsilon} \dot{H}$ is continuous. To show it is also Hölder continuous, we only need to consider points $x_{1}=-a$ and $x_{2}=b$ with $0<a<b$ (if $x_{1} \cdot x_{2} \geq 0$, the sign function remains constant, so $-\sigma B_{Y, \epsilon} \dot{H}$ is in fact $C^{1, \alpha}$ ). Calling $w(x) \equiv-\sigma(x) B_{Y, \epsilon}(x) \dot{H}(x)$ and using that $w(x)$ is even, we find

$$
\begin{array}{r}
\left|w\left(x_{2}\right)-w\left(x_{1}\right)\right|=|w(b)-w(-a)|=\mid\left(w(b)-\left.w(a)|=| \frac{d\left(B_{Y, \epsilon} \dot{H}\right)}{d x}\right|_{x=\zeta}|b-a| \leq\right. \\
\leq\left|\frac{d\left(B_{Y, \epsilon} \dot{H}\right)}{d x}\right|_{x=\zeta}|| b-\left.a\right|^{1-\alpha}\left|x_{2}-x_{1}\right|^{\alpha} \leq\left|\frac{d\left(B_{Y, \epsilon} \dot{H}\right)}{d x}\right|_{x=\zeta}|| x_{2}-\left.x_{1}\right|^{\alpha} .
\end{array}
$$

where $\zeta \in(a, b)$ and we have used that $|b-a|^{\alpha} \leq|b+a|^{\alpha}=\left|x_{2}-x_{1}\right|^{\alpha}$ and $|b-a|<1$. This proves that $-\sigma B_{Y, \epsilon} \dot{H}$ is Hölder continuous with exponent $\alpha$.

To check (ii), we first notice that $w(x)$ obsviously satisfies $\sup _{x}|w|<$ $C\|(H, \delta)\|_{U^{2, \alpha} \times I}$ because $B_{Y, \epsilon}(x)$ is $C^{1, \alpha}$. It remains to bound the Hölder constant $[w]_{\alpha} \equiv \sup _{x_{1} \neq x_{2}} \frac{\left|w\left(x_{2}\right)-w\left(x_{1}\right)\right|}{\left|x_{2}-x_{1}\right|^{\alpha}}$. Combining (A.2) with the fact that $B_{Y, \epsilon}(x)$ is $C^{1, \alpha}$, the bound $[w]_{\alpha} \leq C\|(H, \delta)\|_{U^{2, \alpha} \times I}$ follows at once. This proves (ii) for the term $-\sigma B_{Y, \epsilon} \dot{H}$. A similar argument applies to $-\sigma A_{Y, \epsilon} H$ and $-\sigma C_{Y, \epsilon} \delta$ and we conclude that $D_{Y, \epsilon}|Q|$ is indeed a continuous operator.

In order to apply the implicit function theorem, it is furthermore necessary that $|Q| \in C^{1}\left(U^{2, \alpha} \times I\right)$ (i.e. that $D_{Y, \epsilon}|Q|$ depends continuously on $(Y, \epsilon)$ ). This means that given any convergent sequence $\left(Y_{n}, \epsilon_{n}\right) \in \mathcal{V}_{r_{0}}$, the corresponding operators $D_{Y_{n}, \epsilon_{n}}|Q|$ also converge. Denoting by $(Y, \epsilon) \in \mathcal{V}_{r_{0}}$ the limit of the sequence, we need to prove that $\left\|D_{Y_{n}, \epsilon_{n}}|Q|-D_{Y, \epsilon}|Q|\right\|_{£\left(U^{2, \alpha} \times I, U^{0, \alpha}\right)} \rightarrow 0$. It suffices to find a constant $K$ (which may depend on $(Y, \epsilon))$, such that

$$
\left\|\left(D_{Y_{n}, \epsilon_{n}}|Q|-D_{Y, \epsilon}|Q|\right)(H, \delta)\right\|_{U^{0, \alpha}}<K\|(H, \delta)\|_{U^{2, \alpha} \times I}\left\|\left(Y_{n}-Y, \epsilon_{n}-\epsilon\right)\right\|_{U^{2, \alpha} \times I}
$$

for all $(H, \delta) \in U^{2, \alpha} \times I$. Again, the most difficult case involves $\sigma\left(B_{Y, \epsilon}-B_{Y_{n}, \epsilon_{n}}\right) \dot{H}$, so we concentrate on this term. Using the mean value theorem on the function $\mathcal{B} \equiv \partial_{2} \mathcal{Q}$ (recall that $B_{Y, \epsilon}(x)=\left.\mathcal{B}\right|_{(Y(x), \dot{Y}(x), x, \epsilon)}$ ) gives

$$
\sup _{x}\left|\sigma\left(B_{Y, \epsilon}-B_{Y_{n}, \epsilon_{n}}\right) \dot{H}\right| \leq 2 \sup _{\mathbb{K}}|\nabla \mathcal{B}| \sup _{x}|\dot{H}|\left\|\left(Y_{n}-Y, \epsilon_{n}-\epsilon\right)\right\|_{U^{2, \alpha} \times I},
$$


where $\nabla \mathcal{B}$ is the gradient of $\mathcal{B}$ and $\mathbb{K} \subset \mathbb{R}^{4}$ is a compact domain depending only on $r_{0}$ and $Y_{1}$ defined so that, for all $(Y, \epsilon) \in \mathcal{V}_{r_{0}}$, the quadruple $(Y(x), \dot{Y}(x), x, \epsilon) \in \mathbb{K}$, for all $x \in[-1,1]$. Inequality (A.4) is already of the form (A.3) (recall that $\mathcal{B}$ is smooth). It only remains to bound the Hölder constant of $z \equiv \sigma\left(B_{Y, \epsilon}-B_{Y_{n}, \epsilon_{n}}\right) \dot{H}$ in a similar way. As before, this is done by distinguishing two cases, namely when $x_{1} \cdot x_{2} \geq 0$ and when $x_{1} \cdot x_{2}<0$. Obtaining an inequality of the form $\sup _{x_{1} \neq x_{2}, x_{1} \cdot x_{2} \geq 0} \frac{\left|z\left(x_{2}\right)-z\left(x_{1}\right)\right|}{\left|x_{2}-x_{1}\right|^{\alpha}} \leq$ $K_{1}\|(H, \delta)\|_{U^{2, \alpha} \times I}\left\|\left(Y_{n}-Y, \epsilon_{n}-\epsilon\right)\right\|_{U^{2, \alpha} \times I}$ is standard, because $\sigma(x)$ is a constant function. When $x_{1} \cdot x_{2}<0$, we exploit the parity of the functions as in (A.2) to get $\left|z\left(x_{2}\right)-z\left(x_{1}\right)\right| \leq\left|\frac{d\left(\left(B_{Y_{n}, \epsilon_{n}}-B_{Y, \epsilon}\right) \dot{H}\right)}{d x}\right|_{x=\zeta}\left|x_{2}-x_{1}\right|^{\alpha}$, where $\zeta \in(a, b)$ and we are asumming $x_{1}=-a, x_{2}=b, 0<a<b$ without loss of generality. Bounding the right hand side in terms of $K_{2}\|(H, \delta)\|_{U^{2, \alpha} \times I}\left\|\left(Y_{n}-Y, \epsilon_{n}-\epsilon\right)\right\|_{U^{2, \alpha} \times I}\left|x_{2}-x_{1}\right|^{\alpha}$ is again standard, since the sign function $\sigma(x)$ has already disappeared. This, combined with (A.4) gives (A.3) and hence continuity of the derivative of $D_{Y, \epsilon}|Q|$ with respect to $(Y, \epsilon) \in \mathcal{V}_{r_{0}}$.

The final requirement to apply the implicit function theorem to $F=P-|Q|$ is to check that $\left.D_{Y} F\right|_{\left(Y_{1}, \epsilon=0\right)}$ is invertible. A simple computation gives $\left.D_{Y} F\right|_{\left(Y_{1}, \epsilon=0\right)}(H)=$ $c L(H)$, where $L$ is the elliptic operator defined above, which is an isomorphism between $U^{2, \alpha}$ and $U^{0, \alpha}$. Thus, the implicit function theorem can be used to conclude that there exists an open neighbourhood $\tilde{I} \subset I$ of $\epsilon=0$ and a $C^{1}$ map $\tilde{Y}: \tilde{I} \rightarrow U^{2, \alpha}$ such that $\tilde{Y}(\epsilon=0)=Y_{1}$ and $y=\epsilon \tilde{Y}(\epsilon)$ defines a $C^{2, \alpha}$ generalized apparent horizon embedded in $\Sigma_{\epsilon}$. This proves Proposition 1.

\section{Acknowledgments}

We are very grateful to M. Sánchez for his interest and inestimable help and to H.L. Bray and M. Khuri for useful comments. Financial support under the projects FIS200907238 (Spanish MEC), GR-234 (Junta de Castilla y León) and P09-FQM-4496 (Junta de Andalucía and FEDER funds) are acknowledged. AC acknowledges the Ph.D. grant AP2005-1195 (MEC).

[1] H.L. Bray, M. Khuri, "P.d.e.'s which imply the Penrose conjecture", arXiv:0905.2622 (math.DG).

[2] M. Mars, "Present status of the Penrose inequality", Class. Quantum Grav. 26, 193001 (2009).

[3] R. Penrose, "Naked singularities", Ann. N. Y. Acad. Sci. 224, 125-134 (1973).

[4] L. Andersson, J. Metzger, "The area of horizons and the trapped region", Comm. Math. Phys. 290, 941-972 (2009).

[5] G. Huisken, T. Ilmanen, "The inverse mean curvature flow and the Riemannian Penrose inequality", J. Diff. Geom. 59, 353-437 (2001).

[6] H.L. Bray, "Proof of the Riemannian Penrose inequality using the positive mass theorem", J. Diff. Geom. 59, 177-267 (2001).

[7] K. Karkowski, E. Malec, "The general Penrose inequality: lessons from numerical evidence", Acta Phys. Pol. B36, 59-73 (2005).

[8] P.S. Jang, "On the positivity of energy in general relativity", J. Math. Phys. 19, 1152-1155 (1978).

[9] R. Schoen, S.-Y. Yau, "Proof of the positive mass theorem II", Comm. Math. Phys. 79, 231-260 (1981). 
[10] M. Eichmair, "Existence, Regularity, and Properties of Generalized Apparent Horizons", arXiv: 0805.4454 (math-DG).

[11] M. Khuri, "A note on the nonexistence of generalized apparent horizons in Minkowski space", Class. Quantum Grav. 26, 078001 (2009).

[12] M. Khuri, private communication. 2009.

[13] I. Chavel, "Riemannian geometry, a modern introduction", Cambridge Studies in Advanced Mathematics 98 (Cambridge University Press, 2006).

[14] H.L. Bray, M. Khuri, "A Jang equation approach to the Penrose inequality", arXiv:0910.4785 (math-DG).

[15] P.T. Chruściel, "The classification of static vacuum spacetimes containing an asymptotically flat spacelike hypersurface with compact interior", Class. Quantum Grav. 16, 661-687 (1999). 